\title{
Value of smear and PCR in bronchoalveolar lavage fluid in culture positive pulmonary
} tuberculosis

\author{
C. Tueller*, P.N. Chhajed*, C. Buitrago-Tellez ${ }^{\#}$, R. Frei ${ }^{\dagger}$, M. Frey ${ }^{+}$and M. Tamm*
}

ABSTRACT: At present, further investigations are needed in patients with suspected pulmonary tuberculosis (TB) and either negative sputum smear or without sputum. The aim of the present study was to analyse the yield of bronchoalveolar lavage fluid (BALF) smear and PCR in patients with confirmed pulmonary TB.

Patients with a positive culture for Mycobacterium tuberculosis complex in sputum or BALF were analysed over 5 yrs.

In total, 90 out of 230 (39\%) patients with culture-positive pulmonary TB had a positive sputum smear, and 120 patients underwent bronchoscopy. BALF smear was positive in 56 (47\%), BALF PCR in 93 (78\%) patients, and BALF smear and/or PCR was positive in $83 \%$. In total, 71 patients who underwent bronchoscopy and had complete clinical records were further analysed. BALF (smear or Mycobacterium tuberculosis complex-PCR) allowed a rapid diagnosis in 10 (59\%) out of 17 patients who had a negative sputum smear, and $49(91 \%)$ out of 54 patients without sputum production. Of these 71 patients, 12 (17\%) were only culture positive. Rapid diagnosis of pulmonary TB by smear and/or PCR was made in 190 out of 210 patients (90\%) in sputum or BALF.

In conclusion, combined use of bronchoalveolar lavage fluid smear and Mycobacterium tuberculosis complex-PCR has a good diagnostic yield in patients with sputum smear-negative tuberculosis or without sputum production.

KEYWORDS: Bronchoscopy, culture, PCR, smear, sputum, tuberculosis

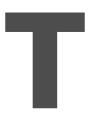

uberculosis (TB) remains a leading health problem in the world, with an estimated 8 million new cases annually, of whom about 3.5 million cases $(44 \%)$ have infectious (smear positive) pulmonary disease $[1,2]$. The global case fatality rate is $\sim 23 \%$ and is $>50 \%$ in some African countries with high HIV rates [1]. Incidences differ between countries, depending on social and economic resources. The prevalence of pulmonary TB in Switzerland is $\sim 10$ cases per 100,000 habitants $\cdot \mathrm{yr}^{-1}$ [3] and lower in people of Swiss origin compared with those who are of non-Swiss origin [4]. The highest incidence of pulmonary TB in Switzerland is observed in refugees and asylum seekers and is estimated to be $>250$ per 100,000 [3].

The investigation for pulmonary TB differs between countries, depending on the prevalence and economic status. In patients from a country with a high prevalence of pulmonary TB and a high clinical suspicion, anti-TB treatment is often initiated without further investigations, even in patients with negative sputum smears
[5]. Ideally, patients with suspected pulmonary TB and one or more smear-negative sputum sample require further investigations to confirm the diagnosis. The diagnosis of "probable" pulmonary TB needs a positive nucleic acid amplification test result or a positive smear, in combination with clinical and radiological findings suggestive of pulmonary TB [6]. A definitive diagnosis of pulmonary $\mathrm{TB}$ needs a positive culture result. In smear-negative specimens, culture growth takes 16-20 days, even when automated and semi-automated liquid culture systems are used [7]. Therefore, further investigations must be undertaken promptly when a patient does not produce sputum spontaneously, or when sputum smear is negative.

Few data exist regarding the combined use of bronchoscopy and PCR in the diagnosis of pulmonary TB. The aim of the present study was to assess the diagnostic yield of bronchoalveolar lavage fluid (BALF) smear and Mycobacterium tuberculosis complex (MTC)-PCR in culture-positive TB and to determine clinical,
AFFILIATIONS

Depts of *Pulmonary Medicine,

\#Radiology, and

'Bacteriology Laboratory, University

Hospital Basel, Basel, and

${ }^{+}$Respiratory Medicine, Hospital

Barmelweid, Barmelweid,

Switzerland

CORRESPONDENCE

P.N. Chhajed

Pulmonary Medicine

University Hospital Basel

Petersgraben 4

4031 Basel

Switzerland

Fax: 41612654587

E-mail: pchhajed@uhbs.ch

Received:

April 182005

Accepted after revision:

July 262005

SUPPORT STATEMENT

None of the authors received financial support for this study. 
radiological and epidemiological characteristics of patients needing bronchoscopy for the diagnosis of pulmonary TB.

\section{PATIENTS AND METHODS}

The current study was conducted at a university hospital in Switzerland located at the border (Basel), which cares for Swiss and non-Swiss inhabitants and is also responsible for further evaluation of asylum seekers and refugees with suspected pulmonary TB screened at the border. All patients identified were registered between January 1997 and June 2003 with a positive culture for Mycobacterium tuberculosis in sputum or BALF. Diagnostic yield of BAL smear and MTC-PCR was calculated for patients who had a negative sputum smear or no sputum at all. Bronchoalveolar lavage was performed as described earlier [8]. All respiratory specimens were digested and decontaminated by the sodium dodecyl sulphate- $\mathrm{NaOH}$ method and then centrifuged. Smears of the sediment were stained with auramine-rhodamine fluorochrome and examined for the presence of acid-fast bacilli by fluorescence microscopy. Positive slides were confirmed by Ziehl-Neelsen staining. Mycobacterial cultures were performed by sample inoculation onto a Loewenstein-Jensen slant (Bio-Rad, Marnesla-Coquette, France) and a Middlebrook 7H10 plus selective 7H11 biplate (Becton Dickinson, Sparks, MD, USA). In addition, processed specimens were inoculated into liquid medium, i.e. a BD BACTEC 12B vial (radiometric Bactec 460-TB system; Becton Dickinson, Franklin Lakes, NJ, USA) by the end of 2000 and since then, a BacT/ALERT MP bottle (BacT/ ALERT 3D system; BioMérieux, Durham, NC, USA). All culture media were incubated for $\geqslant 8$ weeks. For identification of tuberculous mycobacteria, the hybridisation assay AccuProbe culture confirmation kit $M$. tuberculosis complex (Gen-Probe, San Diego, CA, USA) and standard biochemical tests were used. PCR testing for M. tuberculosis complex was performed using the AMPLICOR kits (Roche Diagnostics, Mannheim, Germany) for respiratory specimen preparation, amplification and detection with the COBAS AMPLICOR analyser (Roche Diagnostics, Basel, Switzerland) according to manufacturers' instructions.

The medical records of the patients who underwent bronchoscopy were examined for clinical and laboratory data including HIV status (serological test). Chest radiographs were reviewed by a radiologist blinded to the clinical data. Radiographs were scored as typical of TB when there was a nodular, alveolar or interstitial infiltrate or cavitations predominantly affecting the supraclavicular zones or upper zones of the lung, cavitations in the apical segment of the lower lobe or a miliary pattern. In the absence of these signs, chest radiographs were scored as atypical [9].

\section{Statistics}

Data were analysed using a Fisher's exact test for categorical data and Mann-Whitney U-test for noncategorical data. Data are presented as mean $\pm \mathrm{SD}$. A p-value of $<0.05$ was considered to be statistically significant.

\section{RESULTS}

In total, 230 patients with culture-positive pulmonary TB were identified (fig. 1). In 90 (39\%) patients, the initial diagnosis was based on sputum smear, and in 20 (9\%) patients sputum smear was negative, but no further investigations were performed.

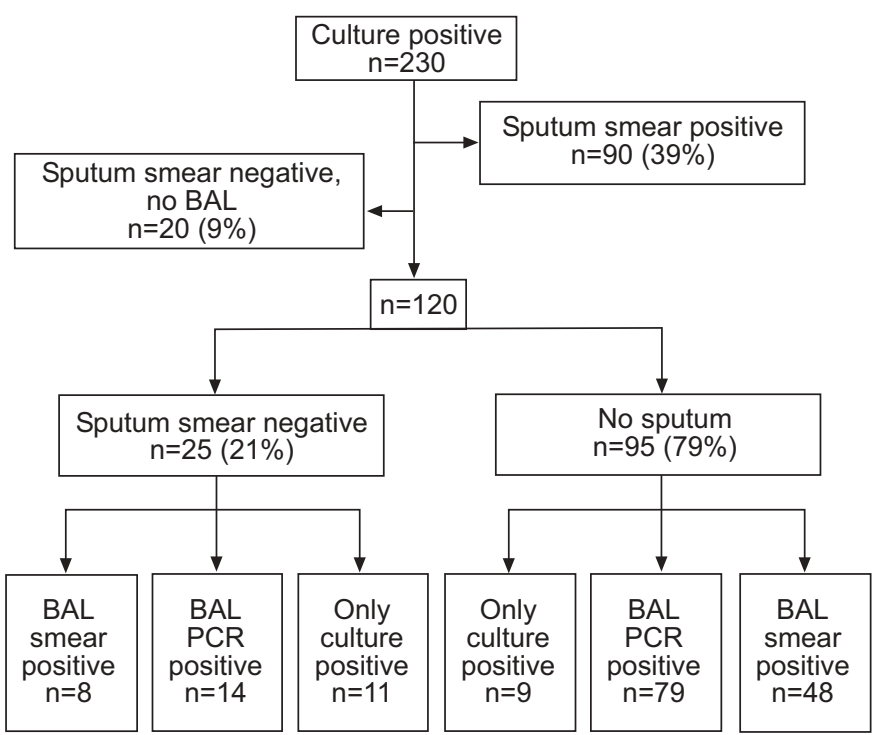

FIGURE 1. Analysis of patients with a positive culture for Mycobacterium tuberculosis. BAL: bronchoalveolar lavage.

Bronchoscopy with bronchoalveolar lavage was performed in $120(52 \%)$ patients. The diagnostic yield of BALF direct smear for TB was $47 \%$ (56 out of 120) and BALF MTC-PCR was positive in $78 \%$ (93 out of 120) of patients. BALF MTC-PCR was exclusively positive in 44 patients who were BALF smear negative. Therefore, the overall diagnostic yield of BALF was $83 \%$ (100 out of 120$)$. Of the 25 patients with a negative sputum smear, BALF led to rapid diagnosis in $14(56 \%)$ patients (eight BAL MTC-PCR and BALF smear positive; six BALF MTC-PCR positive and BALF smear negative), and 11 (44\%) were only culture positive. Of the 95 patients, 86 (91\%) who did not produce sputum spontaneously were rapidly diagnosed by BALF (smear and/or MTC-PCR positive). Of the 120 patients, $20(17 \%)$ with smear-negative sputum or no sputum were only culture positive. Combining sputum smear and BALF smear/ MTC-PCR, a rapid diagnosis of pulmonary TB was made in 190 of $210(90 \%)$ patients.

In 49 out of 120 patients who underwent bronchoscopy, medical records were not available or incomplete, and these patients were excluded from further analysis. Therefore, clinical data from the remaining 71 patients who underwent BALF for diagnosis of pulmonary TB could be analysed in detail. Of these, 17 (24\%) patients had a negative sputum smear and $54(76 \%)$ patients had no sputum production, $55(77 \%)$ were males and $44(62 \%)$ were smokers. In total, there were 27 (38\%) patients of Swiss origin (aged $49 \pm 16 \mathrm{yrs}), 16$ (23\%) from Southern Europe (aged 38 \pm 18 yrs), 11 (15\%) from Eastern Europe and Eastern Mediterranean (aged $32 \pm 10 \mathrm{yrs}$ ) and 17 $(24 \%)$ from other countries (Africa $(n=8)$, Asia $(n=4)$, South America $(n=3)$ and Middle East $(n=2)$; aged $33 \pm 15$ yrs $)$. Only three $(4 \%)$ of these patients had a known history of TB. Comorbidities, such as alcohol abuse (16 patients) and diabetes (three patients), were present in 19 patients. Four patients had a history of both alcohol and intravenous drug abuse. History of diabetes or alcohol abuse was present in 14 out of $27(52 \%)$ patients of Swiss origin and in five out of $44(11 \%)$ patients of 
non-Swiss origin. Serological testing for HIV was positive in six $(8 \%)$ patients, negative in 41 patients and has not been performed in 24 patients.

\section{Clinical and radiological findings}

Symptoms (cough, weight loss, night sweats, fever, haemoptysis, dyspnoea) were present in 58 out of $71(82 \%)$ patients. Twelve $(17 \%)$ patients were completely asymptomatic. Ten patients were suspected to have pulmonary TB based on chest radiograph screening performed for asylum seekers, and the remaining two patients had pulmonary infiltrates of unknown cause. In one patient, symptoms were not registered. The most frequent symptom was cough $(69 \%)$, followed by weight loss $(43 \%)$, night sweats $(31 \%)$, fever $(27 \%)$, dyspnoea $(12 \%)$ and haemoptysis $(11 \%)$. Forty-seven $(66 \%)$ patients had two or more symptoms. Chest radiographs were available for review in 57 patients with $41(71 \%)$ scored as typical for TB with cavitations in 14 patients. Two patients had normal chest radiographs, and the reasons for bronchoscopy were typical symptoms as described above.

\section{C-reactive protein and white blood cell count}

Mean C-reactive protein (CRP) was $45 \pm 67 \mathrm{mg} \cdot \mathrm{L}^{-1}$ (range 1$\left.399 \mathrm{mg} \cdot \mathrm{L}^{-1}\right)$. Twenty $(28 \%)$ patients had a CRP $>50 \mathrm{mg} \cdot \mathrm{L}^{-1}$ and $18(90 \%)$ of them were symptomatic. Asymptomatic patients had a mean CRP of $15 \pm 17 \mathrm{mg} \cdot \mathrm{L}^{-1}$ and a mean white blood cell count of $7.6 \pm 2.6 \times 10^{9} \cdot \mathrm{L}^{-1}$. The mean white blood cell count of all patients was $8.5 \pm 3.7 \times 10^{9} \cdot \mathrm{L}^{-1}\left(\right.$ range $\left.4.2-25 \times 10^{9} \cdot \mathrm{L}^{-1}\right)$. Only 16 out of $71(22 \%)$ patients had a white blood cell count $>10 \times 10^{9} \cdot \mathrm{L}^{-1}$, and, of those, $14(87 \%)$ were symptomatic.

\section{Clinical data based on the prevalence of TB based on country of birth}

Of the study patients, 31 were from countries with a prevalence of TB $<10$ per 100,000 (Switzerland $(n=27)$ and four from Italy $(n=4))$. Nine patients were from countries with a prevalence of 10-50 per 100,000 (Spain $(n=1)$, Albania $(n=2)$, and Turkey $(n=6))$. The remaining 31 patients were from countries with a prevalence of $>50$ per 100,000 (former Yugoslavia $(n=8)$, Georgia $(n=3)$, Brazil, Sierra Leone and Iraq (each $\mathrm{n}=2$ ), and Croatia, Armenia, Algeria, Russia, Thailand, Peru, China, Angola, India, Pakistan, Senegal, Liberia, Ethopia and Nigeria $($ each $n=1)$ ) [10]. Based on the American Thoracic Society and Centers for Disease Control and Prevention guidelines, the clinical data was analysed to assess the pre-test probability of TB by examining whether the patients belonged to the higher prevalence groups and/or high-risk groups (table 1) [11]. The mean age, smoking history and the presence of two or more symptoms were significantly higher in the low-prevalence group $(<10$ per 100,000) compared with the other two groups (table $1 ; \mathrm{p}<0.05$ ). Alcohol abuse was also significantly higher in patients belonging to the low-prevalence group $(<10$ per 100,000$)$ compared with the high-prevalence group $(>50$ per 100,000 ; $p>0.05$; table 1). The finding of a typical chest radiograph was significantly higher in patients belonging to the high prevalence group $(>50$ per 100,000$)$ compared with the lowprevalence group $(<10$ per 100,$000 ; \mathrm{p}<0.05$; table 1$)$.

Endobronchial abnormalities were seen in 18 (25\%) patients, including acute inflammation $(n=10)$, purulent bronchitis

\begin{tabular}{|c|c|c|c|}
\hline $\begin{array}{l}\text { Clinical d } \\
\text { of tuberc }\end{array}$ & $\begin{array}{l}\text { a based ol } \\
\text { sis (TB) b }\end{array}$ & $\begin{array}{l}\text { evalence } \\
\text { ountry of } k\end{array}$ & $100,000)$ \\
\hline & $\begin{array}{l}<10 \text { per } \\
100,000\end{array}$ & $\begin{array}{c}10-50 \text { per } \\
100,000\end{array}$ & $\begin{array}{l}>50 \text { per } \\
100,000\end{array}$ \\
\hline Subjects $n$ & 31 & 9 & 31 \\
\hline Age yrs & $50 \pm 18$ & $31 \pm 12$ & $33 \pm 13$ \\
\hline Smoking history & $25(81)$ & $3(33)$ & $16(52)$ \\
\hline Previous TB & Nil & $2(22)$ & $1(3)$ \\
\hline HIV & $5 / 25(20)$ & Nil & $1 / 20(5)$ \\
\hline$\geqslant 2$ symptoms & $25(81)$ & $4(44)$ & $17(55)$ \\
\hline Alcohol abuse & $13(42)$ & $\mathrm{Nil}$ & $3 / 31(9)$ \\
\hline Diabetes mellitus & $4(13)$ & Nil & $2 / 31(6)$ \\
\hline Intravenous drug abuse & $3(10)$ & Nil & $1 / 31(3)$ \\
\hline Typical chest radiograph & $13 / 24(54)$ & 6/7 (86) & $21 / 25(84)$ \\
\hline CRP & $34(5-399)$ & $14(5-53)$ & $6(1-140)$ \\
\hline $\begin{array}{l}\text { White blood cell count } \\
\times 10^{9} \cdot \mathrm{L}^{-1}\end{array}$ & $9.3 \pm 4.6$ & $7.3 \pm 1.3$ & $8.0 \pm 3.0$ \\
\hline
\end{tabular}

Data are presented as $n$, mean $\pm \mathrm{SD}, \mathrm{n}(\%)$ or median (range). CRP: C-reactive protein.

$(n=4)$, hemorrhagic bronchitis $n=3)$ and perforated caseous lymph nodes $(n=1)$.

Bacteria were grown in BALF culture in 16 out of $71(22 \%)$ patients. The bacteria found were: Staphylococcus pneumoniae $(\mathrm{n}=5)$, Haemophilus influenzae $(\mathrm{n}=5)$, Enterobacter spp. $(\mathrm{n}=2)$, Neisseria spp. $(\mathrm{n}=3)$ and Klebsiella spp. $(\mathrm{n}=1)$. Drug resistant TB was present in $10(15 \%)$ patients, with four of them showing multidrug resistance.

The comparative data for patients who had a rapid diagnostic bronchoscopy (BALF smear or MTC-PCR positive; 59 out of 71 patients) and those in whom the definitive diagnosis was delayed (BALF smear and MTC-PCR negative, but culture positive; 12 out of 71 patients) are presented in table 2 . The two groups did not differ in regard to epidemiological characteristics and radiological findings. However, patients in whom the definitive diagnosis was delayed (only BALF culture positive) were significantly less symptomatic and had a significantly lower CRP and white blood cell count.

\section{DISCUSSION}

The diagnosis of TB based on a positive sputum smear is reported in $22-50 \%$ of patients with culture-positive pulmonary TB $[1,12-15]$. The $39 \%$ diagnostic rate of sputum smear in the current authors' institution is in the same range as reported in literature.

The value of MTC-PCR in respiratory samples to obtain a rapid diagnosis of pulmonary TB has been described in many studies [16-18]. In smear-positive specimens MTC-PCR has a sensitivity of $86-95 \%$ and specificity of $98-100 \%$, whereas in smearnegative specimens, the sensitivity is significantly lower at $62-77 \%$, while the specificity is still high at 98\% [18-20]. MTCPCR of bronchial aspirates in patients with smear-negative sputum has a sensitivity reported ranging 36-97\% [21, 22]. This wide range might be explained by the use of different samples (BALF versus bronchial secretions) and differences in patient 


\begin{tabular}{|c|c|c|c|c|}
\hline \multirow[t]{2}{*}{ TABLE 2} & \multicolumn{4}{|c|}{$\begin{array}{l}\text { Characteristics of patients with a rapidly } \\
\text { diagnostic bronchoalveolar lavage (BAL) } \\
\text { compared to those with a delayed diagnosis with } \\
\text { only a positive culture }\end{array}$} \\
\hline & & $\begin{array}{c}\text { BAL smear } \\
\text { or PCR positive }\end{array}$ & $\begin{array}{l}\text { Only culture } \\
\text { positive }\end{array}$ & p-value \\
\hline \multicolumn{2}{|l|}{ Subjects $\mathbf{n}$} & 59 & 12 & \\
\hline \multicolumn{2}{|l|}{ Age yrs } & $41 \pm 18$ & $38 \pm 14$ & 0.7 \\
\hline \multicolumn{2}{|l|}{ Males } & $45(76)$ & $10(83)$ & 0.27 \\
\hline \multicolumn{2}{|l|}{ Smokers } & $36(61)$ & $7(58)$ & 0.25 \\
\hline \multicolumn{2}{|l|}{ HIV positive } & $5(8)$ & $1(8)$ & 0.42 \\
\hline \multicolumn{2}{|l|}{ Swiss origin } & $24(41)$ & $3(25)$ & 0.16 \\
\hline \multicolumn{2}{|c|}{ Typical chest radiograph } & $33 / 48(69)^{\#}$ & $7 / 8(87)^{\#}$ & 0.21 \\
\hline \multicolumn{2}{|c|}{ Radiological cavitations } & $13 / 48(27)^{\#}$ & $1 / 8(12)^{\#}$ & 0.27 \\
\hline \multicolumn{2}{|c|}{ No symptoms } & $8(14)$ & $4(33)$ & 0.09 \\
\hline \multicolumn{2}{|c|}{$\geqslant 2$ symptoms } & $41(70)$ & $6(50)$ & 0.02 \\
\hline \multicolumn{2}{|c|}{ CRP $\mathrm{mg} \cdot \mathrm{L}^{-1}$} & $22(0.5-138)$ & $5(3-399)$ & $<0.001$ \\
\hline \multicolumn{2}{|c|}{$\begin{array}{l}\text { White blood cell count } \\
\times 10^{9} \cdot \mathrm{L}^{-1}\end{array}$} & $8.9 \pm 4.0$ & $6.5 \pm 1.2$ & 0.009 \\
\hline
\end{tabular}

Data are presented as $\mathrm{n}$, mean $\pm \mathrm{SD}, \mathrm{n}(\%)$ or median (range). CRP: C-reactive protein. ${ }^{\# \text { : }}$ some patients were unavailable.

characteristics (patients with clinical symptoms of TB versus patients with only radiographical abnormalities). MTC-PCR in BALF had a sensitivity of $78 \%$ for detection of pulmonary TB in sputum smear-negative patients or patients without sputum production. The combination of BALF smear and MTC-PCR has a very high diagnostic yield of $91 \%$ in patients not producing sputum and of $56 \%$ in patients with smear-negative sputum.

Several studies have compared induced sputum with bronchoscopy for the diagnosis of pulmonary TB. Induced sputum is reported to produce a positive smear result in $22-64 \%$ of patients with suspected pulmonary $\mathrm{TB}$ who do not produce sputum or who have smear-negative spontaneous sputum [2325]. This is less compared with the present results with BALF MTC-PCR. Studies directly comparing sputum induction with bronchoscopy in low-prevalence countries using nucleic acid amplification techniques for rapid diagnosis of pulmonary TB are, to the current authors' knowledge, not available. This aspect could not be assessed in the present study, as sputum induction is not performed routinely in the hospital. However, sputum induction is less expensive than bronchoscopy and has been reported to have a comparable diagnostic yield to bronchoscopy when three induced sputa are analysed [13, 25, 26]. In these studies, sputum induction with hypertonic saline produced an adequate specimen in $71-99 \%$ of patients. The findings of the current study suggest that in a country with a low prevalence of TB, BALF smear and MTC-PCR allows a rapid diagnosis of $\mathrm{TB}$ in $>80 \%$ of patients who are either sputum smear negative or not able to produce sputum. Therefore, a final diagnosis can be made within 1 or 2 days.

Male dominance and the high incidence of smoking history in almost two thirds of the patients concur with observations reported in the literature [27]. The rate of Swiss patients and foreigners with pulmonary $\mathrm{TB}$ (38 and 62\%, respectively) is within the range reported in a recent epidemiological survey of TB in Switzerland [4]. Bronchoscopy, for reasons other than for suspicion of pulmonary $\mathrm{TB}$, such as haemoptysis or radiological undetermined infiltrates, was performed in one fifth of patients. As pulmonary TB has a low prevalence in Switzerland, treating physicians do not often consider TB as a differential diagnosis. Alcohol abuse and diabetes were much more common in pulmonary TB patients of Swiss origin compared to non-Swiss patients. This is in accordance with an Italian study that found alcohol abuse, diabetes and history of recent contact to be the most important risk factors for pulmonary TB among native Italians [15]. Therefore, pulmonary TB should be considered as a differential diagnosis in patients with alcohol abuse and diabetes who have radiological infiltrates.

Most of the study patients with culture-proven pulmonary TB had typical abnormalities on chest radiographs, which is consistent with the literature [9, 28, 29]. Atypical patterns are often found in patients with diabetes or in renal transplant recipients [30]. However, REICHENBERGER et al. [31] have previously reported a very low incidence of TB in immunocompromised patients. Between 42 and $86 \%$ of patients with pulmonary TB may be symptomatic $[32,33]$. Only $20 \%$ of the study patients with confirmed pulmonary TB were completely asymptomatic. Asymptomatic patients had a lower CRP level, whereas white blood cell counts were in the same range in both symptomatic and asymptomatic patients. Of the 12 asymptomatic patients in the study, nine were asylum seekers screened at the border by chest radiographs, and eight of those had lesions typical for pulmonary TB. Patients with a diagnosis of pulmonary TB only in culture (sputum and BALF smear and MTC-PCR negative) usually have lower CRP and white blood cell counts compared with patients who are BALF smear or MTC-PCR positive. Furthermore, they are less likely to have two or more respiratory or constitutional symptoms.

The pre-test evaluation is based on the high prevalence and high risk-factor grouping. This suggests that patients with culture-positive TB, from low-prevalence countries, had a lesser chance of presenting with a typical chest radiograph suggestive of $\mathrm{TB}$, but had more constitutional symptoms. However, those patients from high-prevalence countries had a significantly higher chance of presenting with a typical chest radiograph suggestive of $\mathrm{TB}$, but had lesser constitutional symptoms compared with patients from low-prevalence states. Therefore, the findings of the present study suggest that bronchoscopy is useful in patients from countries with low prevalence as well as high prevalence of TB (table 1). Physicians might be aware of a potential diagnosis of TB in patients originating from a high-prevalence country, which might explain the differences in the clinical presentation. It also highlights that physicians should always think about TB in HIV-negative patients from low-prevalence countries.

Gastric aspiration is a valuable method in the diagnosis of TB in children. It has also been used in the diagnosis of pulmonary TB for $>30$ yrs in adults, but has not found a place in routine clinical use [34]. Bronchial washings have been reported to be superior to gastric washings in making a culture-positive diagnosis of TB [35]. Recently, the string test has been documented to be safe and effective for the retrieval of useful 
clinical specimens for diagnosis of pulmonary TB [36] However, to the authors' knowledge, there are no data comparing the string test with BALF.

The limitations of the current study are its retrospective nature and the unavailability of induced sputum. The value of induced sputum for sputum smear-negative tuberculosis is currently under discussion $[13,25,26]$. Induced sputum has been shown to be at least as good as bronchoscopy when molecular methods are not used to achieve a rapid diagnosis. However, randomised controlled trials comparing three induced sputa using smear, Mycobacterium tuberculosis complex-PCR and culture, with bronchoalveolar lavage fluid smear, Mycobacterium tuberculosis complex-PCR and culture are lacking. Therefore, the current findings support that combining bronchoalveolar lavage fluid smear and Mycobacterium tuberculosis complex-PCR allow for a rapid diagnosis of pulmonary tuberculosis in patients who are sputum smear negative or have no sputum production at all.

\section{REFERENCES}

1 Dye C, Scheele S, Dolin P, Pathania V, Raviglione MC. Consensus statement. Global burden of tuberculosis: estimated incidence, prevalence, and mortality by country. WHO Global Surveillance and Monitoring Project. JAMA 1999; 282: 677-686.

2 Treatment of Tuberculosis: Guidelines for National Programmes. Geneva, World Health Organisation, 1997. Report No. WHO document WHO/TB/97.220.

3 Maurer AM, Seiler AJ. Diagnosis and treatment of pulmonary tuberculosis in asylum seekers and refugees in the Bern canton 1993-1997. Schweiz Rundsch Med Praxis 2000; 89: 271-274.

4 Tuberculosis in Switzerland: 1999 to 2000. Bundesamt für Gesundheitswesen, Bulletin 2002, No. 9, 25.2.2002; pp. 168-174.

5 Mohan A, Pande JN, Sharma SK, Rattan A, Guleria R, Khilnani GC. Bronchoalveolar lavage in pulmonary tuberculosis: a decision analysis approach. QJM 1995; 88: 269-276.

6 Brändli O, Prodhom G, Rochat T, Zellweger JP. Clinic and diagnosis of tuberculosis. Schweiz Med Forum 2003; 21: 492-497.

7 Watterson SA, Drobniewski FA. Modern laboratory diagnosis of mycobacterial infections. J Clin Pathol 2000; 53: 727-732.

8 Tamm M, Traenkle P, Grilli B, et al. Pulmonary cytomegalovirus infection in immunocompromised patients. Chest 2001; 119: 838-843.

9 Tattevin P, Casalino E, Fleury L, Egmann G, Ruel M, Bouvet E. The validity of medical history, classic symptoms, and chest radiographs in predicting pulmonary tuberculosis: derivation of a pulmonary tuberculosis prediction model. Chest 1999; 115: 1248-1253.

10 Tuberculosis prevalence rate per 100,000 population (WHO). United Nations Statistics Division, 2005. Report No. 29982.

11 Diagnostic Standards and Classification of Tuberculosis in Adults and Children. This official statement of the American Thoracic Society and the Centers for Disease
Control and Prevention was adopted by the ATS Board of Directors, July 1999. This statement was endorsed by the Council of the Infectious Disease Society of America, September 1999. Am J Respir Crit Care Med 2000; 161: 1376-1395.

12 Valadas E, Hanscheid T, Fernandes ML, Antunes F. Smear microscopy to diagnose tuberculosis early and prevent further transmission in a population with a high prevalence of HIV infection. Clin Microbiol Infect 2003; 9: 1045-1047.

13 Conde MB, Soares SL, Mello FC, et al. Comparison of sputum induction with fiberoptic bronchoscopy in the diagnosis of tuberculosis: experience at an acquired immune deficiency syndrome reference center in Rio de Janeiro, Brazil. Am J Respir Crit Care Med 2000; 162: 2238-2240.

14 Mehta J, Krish G, Berro E, Harvill L. Fiberoptic bronchoscopy in the diagnosis of pulmonary tuberculosis. South Med J 1990; 83: 753-755.

15 Centis R, Migliori GB. Evaluation of tuberculosis treatment results in Italy, report 1999. Monaldi Arch Chest Dis 2002; 57: 297-305.

16 Yoon KH, Cho SN, Lee TY, et al. Detection of Mycobacterium tuberculosis in clinical samples from patients with tuberculosis or other pulmonary diseases by polymerase chain reaction. Yonsei Med J 1992; 33: 209-216.

17 Querol JM, Farga MA, Granda D, Gimeno C, Garcia-deLomas J. The utility of polymerase chain reaction (PCR) in the diagnosis of pulmonary tuberculosis. Chest 1995; 107: 1631-1635.

18 Su WJ, Tsou AP, Yang MH, Huang CY, Perng RP. Clinical experience in using polymerase chain reaction for rapid diagnosis of pulmonary tuberculosis. Zhonghua Yi Xue Za Zhi (Taipei) 2000; 63: 521-526.

19 Clarridge JE 3rd, Shawar RM, Shinnick TM, Plikaytis BB. Large-scale use of polymerase chain reaction for detection of Mycobacterium tuberculosis in a routine mycobacteriology laboratory. J Clin Microbiol 1993; 31: 2049-2056.

20 Bogard M, Vincelette J, Antinozzi R, et al. Multicenter study of a commercial, automated polymerase chain reaction system for the rapid detection of Mycobacterium tuberculosis in respiratory specimens in routine clinical practice. Eur J Clin Microbiol Infect Dis 2001; 20: 724-731.

21 Chen NH, Liu YC, Tsao TC, et al. Combined bronchoalveolar lavage and polymerase chain reaction in the diagnosis of pulmonary tuberculosis in smear-negative patients. Int J Tuberc Lung Dis 2002; 6: 350-355.

22 Wong CF, Yew WW, Chan CY, Au LY, Cheung SW, Cheng AF. Rapid diagnosis of smear-negative pulmonary tuberculosis via fibreoptic bronchoscopy: utility of polymerase chain reaction in bronchial aspirates as an adjunct to transbronchial biopsies. Respir Med 1998; 92: 815-819.

23 Parry CM, Kamoto O, Harries AD, et al. The use of sputum induction for establishing a diagnosis in patients with suspected pulmonary tuberculosis in Malawi. Tuber Lung Dis 1995; 76: 72-76.

$24 \mathrm{Li} \mathrm{LM}$, Bai LQ, Yang HL, et al. Sputum induction to improve the diagnostic yield in patients with suspected pulmonary tuberculosis. Int J Tuberc Lung Dis 1999; 3: 1137-1139. 
25 Al Zahrani K, Al Jahdali H, Poirier L, Rene P, Menzies D. Yield of smear, culture and amplification tests from repeated sputum induction for the diagnosis of pulmonary tuberculosis. Int J Tuberc Lung Dis 2001; 5: 855-860.

26 Anderson C, Inhaber N, Menzies D. Comparison of sputum induction with fiber-optic bronchoscopy in the diagnosis of tuberculosis. Am J Respir Crit Care Med 1995; 152: 1570-1574.

27 Kolappan C, Gopi PG. Tobacco smoking and pulmonary tuberculosis. Thorax 2002; 57: 964-966.

28 Kanaya AM, Glidden DV, Chambers HF. Identifying pulmonary tuberculosis in patients with negative sputum smear results. Chest 2001; 120: 349-355.

29 Wilcke JT, Askgaard DS, Nybo Jensen B, Dossing M. Radiographic spectrum of adult pulmonary tuberculosis in a developed country. Respir Med 1998; 92: 493-497.

30 Chang GC, Wu CL, Pan SH, et al. The diagnosis of pneumonia in renal transplant recipients using invasive and noninvasive procedures. Chest 2004; 125: 541-547.
31 Reichenberger F, Dickenmann M, Binet I, et al. Diagnostic yield of bronchoalveolar lavage following renal transplantation. Transpl Infect Dis 2001; 3: 2-7.

32 MariottaS, MasulloM,Guidi L, AquiliniM,Pabani R, Bisetti A. Tracheobronchial involvement in 84 cases of pulmonary tuberculosis. Monaldi Arch Chest Dis 1995; 50: 356-359.

33 McWilliams T, Wells AU, Harrison AC, Lindstrom S, Cameron RJ, Foskin E. Induced sputum and bronchoscopy in the diagnosis of pulmonary tuberculosis. Thorax 2002; 57: 1010-1014.

34 Elliott RC, Reichel J. The efficacy of sputum specimens obtained by nebulization versus gastric aspirates in the bacteriologic diagnosis of pulmonary tuberculosis. A comparative study. Am Rev Respir Dis 1963; 88: 223-227.

35 Rizvi N, Rao NA, Hussain M. Yield of gastric lavage and bronchial wash in pulmonary tuberculosis. Int $J$ Tuberc Lung Dis 2000; 4: 147-151.

36 Vargas D, Garcia L, Gilman RH, et al. Diagnosis of sputumscarce HIV-associated pulmonary tuberculosis in Lima, Peru. Lancet 2005; 365: 150-152. 